\title{
Enzyme-Like Catalysis of the Nazarov Cyclization by Supramolecular Encapsulation
}

\author{
Courtney J. Hastings, Michael D. Pluth, Robert G. Bergman, ${ }^{*}$ Kenneth N. Raymond*
}

\author{
Department of Chemistry, University of California, Berkeley, California, and Division of Chemical Sciences, Lawrence \\ Berkeley National Laboratory, Berkeley, California, 94720-1460
}

\section{RECEIVED DATE (automatically inserted by publisher); Email: rbergman@berkeley.edu; raymond@socrates.berkeley.edu}

A primary goal in the design and synthesis of molecular hosts has been the selective recognition and binding of a variety of guests using non-covalent interactions. ${ }^{1}$ Supramolecular catalysis, which is the application of such hosts towards catalysis, ${ }^{2}$ has much in common with many enzymatic reactions, ${ }^{3}$ chiefly the use of both spatially appropriate binding pockets and precisely oriented functional groups to recognize and activate specific substrate molecules. Although there are now many examples which demonstrate how selective encapsulation in a host cavity can enhance the reactivity of a bound guest, all have failed to reach the degree of increased reactivity typical of enzymes. ${ }^{4}$ We now report the catalysis of the Nazarov cyclization by a self-assembled coordination cage, a carbon-carbon bond-forming reaction which proceeds under mild, aqueous conditions. The acceleration in this system is over a million-fold, and represents the first example of supramolecular catalysis that achieves the level of rate enhancement comparable to that observed in several enzymes. We explain the unprecedented degree of rate increase as due to the combination of (a) preorganization of the encapsulated substrate molecule, (b) stabilization of the transition state of the cyclization by constrictive binding, and (c) increase in the basicity of the complexed alcohol functionality.

Raymond and coworkers have designed a supramolecular host (1) with $\mathrm{Ga}_{4} \mathrm{~L}_{6}$ stoichiometry ( $\mathrm{L}=$ N,N -bis(2,3dihydroxybenzoyl)-1,5-diaminonaphthalene, Figure 1) that exploits reversible metal-ligand interactions to spontaneously self-assemble. ${ }^{5}$ In these assemblies the biscatecholate ligands span the edges of a tetrahedron in which the vertices are occupied by metal ions. Polyanion $\mathbf{1}$ is soluble in water and other polar solvents, while the host interior is panelled by the naphthalene rings of the ligand, creating a hydrophobic inner environment. A wide variety of cationic guests are encapsulated in $\mathbf{1}$, from quaternary ammonium and phosphonium cations to organometallic complexes. In aqueous solution, neutral molecules, such as hydrocarbons, are bound by 1 due to the hydrophobic effect. ${ }^{6}$

Our approach towards supramolecular catalysis using $\mathbf{1}$ exploits the polyanionic host's preference for encapsulating monocationic guests. Encapsulation in $\mathbf{1}$ can perturb acid-base equilibria to favor the protonation of a wide range of amines and phosphines, even at strongly basic $\mathrm{pH}^{7}$ These investigations led to the development of proton-catalyzed hydrolysis reactions inside of $\mathbf{1}$, in which a protonated transition-state is stabilized in the host interior. ${ }^{8}$ The stabilization of even transient protonated species produces a several thousandfold rate acceleration of orthoformate and acetal hydrolysis under basic conditions. However, even this substantial acceleration does not reach that typically seen in enzyme-catalyzed reactions.
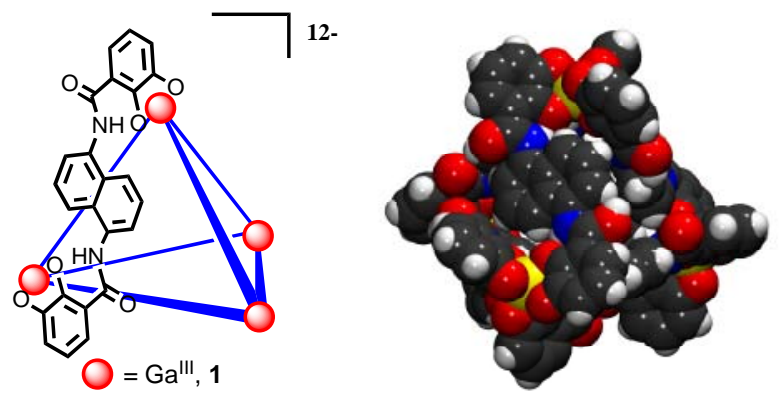

Figure 1. Left: A schematic view of $\mathbf{1}$, where the bis-bidentate ligands are represented by blue lines and the gallium atoms are represented by red circles. Right: A space-filling model of $\mathbf{1}$.

Having demonstrated the ability of $\mathbf{1}$ to perform acidcatalyzed hydrolysis reactions, we sought to apply this reactivity to more synthetically useful acid-catalyzed reaction types. Acid-catalyzed cyclizations are particularly interesting because 1 could accelerate such a reaction both by enhancing the basicity of the bound substrate, and by preorganizing the substrate in a reactive conformation. ${ }^{9}$ The Nazarov cyclization, an acid-catalyzed reaction in which a 1,4-dien-3ol forms a cyclopentadiene (e.g., the example shown in Scheme 2a) is attractive from this perspective. This reaction proceeds via the intermediacy of a diallylic carbocation, which undergoes a conrotatory electrocyclic ring closure in accordance with the Woodward-Hoffman rules. ${ }^{10}$ This reaction is widely utilized in synthetic organic chemistry, as well as in organometallic chemistry where it has provided a route to substituted polymethylcyclopentadienyl ligands. ${ }^{11}$

As proof of principle, 10 equivalents of 3,4,5trimethylhepta-2,5-dien-4-ol 2 (obtained as a mixture of the three possible olefin stereoisomers, Scheme $2 \mathrm{~b}$ ), were added to a solution of 1 in $\mathrm{H}_{2} \mathrm{O}$ (buffered to $\mathrm{pH}$ 11.0), and heated at $50{ }^{\circ} \mathrm{C}$. Under these reaction conditions a set of peaks were observed by ${ }^{1} \mathrm{H}$-NMR, corresponding to host-guest complexes of 2. After twelve hours, the organic products were extracted into $\mathrm{CH}_{2} \mathrm{Cl}_{2}$, whereupon GC-MS analysis showed the complete consumption of the starting material and the quantitative formation of the Nazarov product, Cp*H. A control reaction run using 1.1 equivalents of a strongly binding guest $\left(\mathrm{NEt}_{4}{ }^{+}\right)$to block the host interior halted product 
formation, through a mechanism analogous to enzyme inhibition. $^{12}$

Scheme 1: (a) General scheme for the acid-catalyzed Nazarov reaction of pentadienols to form cyclopentadienes. (b) Pentadienol reactants for the Nazarov cyclization used in this study.

a

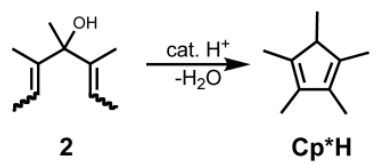

b

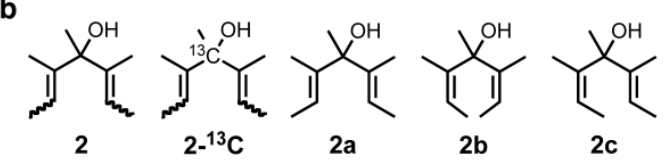

Having obtained these preliminary results, we sought to quantify the rate enhancement of the catalyzed reaction over the background reaction. Due to the low solubility of substrate 2 in water, further studies were carried out in $\mathrm{D}_{2} \mathrm{O}$ with $50 \%$ added DMSO- $\mathrm{d}_{6}$. The three possible stereoisomers of 2 (Scheme 2b) were independently synthesized for these experiments. Under these conditions the disappearance of each of the three stereoisomers of 2 catalyzed by $7 \mathrm{~mol} \% \mathbf{1}$ were monitored by ${ }^{1} \mathrm{H}$-NMR spectroscopy. The product of these reactions, $\mathrm{Cp} * \mathrm{H}$, is not soluble under these conditions and is not observed during the course of the reaction, but can be extracted into organic solvent after the reaction is complete. Again, no reaction was observed when a strongly-binding guest was added to exclude the substrate from the interior of $\mathbf{1}$.

For all three stereoisomers of $\mathbf{2}$, the initial reactions were rapid, but a deviation from first-order kinetic behavior was seen as the reaction progressed. In each case the $k_{\mathrm{obs}}$ of starting material disappearance (calculated from the natural log plot of concentration versus time) was constant at the beginning of the reaction, and then decreased as the reaction proceeded. This effect was especially severe for the reaction of substrate 2a, which nearly halted after $25 \%$ conversion. This decrease in reaction rate is consistent with product inhibition, a common occurrence in both synthetic and enzymatic catalysis when the host does not bind the reactant substantially more strongly than it binds the product. ${ }^{13}$ Competition experiments between $\mathrm{Cp} * \mathrm{H}$ and the stereoisomers of $\mathbf{2}$ show that $\mathrm{Cp}^{*} \mathrm{H}$ is a competitive guest. Adding a full equivalent of $\mathrm{Cp}^{*} \mathrm{H}$ to the 1-catalyzed reaction shut down the Nazarov cyclization. These experiments clearly implicate product inhibition as the cause of the decrease of $k_{o b s}$ that is observed as the reaction progresses.

A solution to this problem was developed by chemically converting the product $\mathrm{Cp} * \mathrm{H}$ into a poor guest, a strategy that has been utilized in other examples of supramolecular catalysis. The addition of maleimide (3) to the 1-catalyzed Nazarov cyclization completely alleviates product inhibition by converting $\mathrm{Cp} * \mathrm{H}$ into the Diels-Alder adduct 4 , which is soluble under the reaction conditions (Scheme 2). In the reaction using maleimide as a trapping agent, the rate of DielsAlder adduct formation is equal to rate of starting material consumption, and implies that no $\mathrm{Cp}^{*} \mathrm{H}$ builds up in solution. The concentration versus time plots for reactions with added maleimide subsequently showed no deviation from first order behavior, indicating that product inhibition was eliminated. Competitive binding experiments between $\mathrm{Cp}^{*} \mathrm{H}$ and the Diels-Alder adduct (4) showed that $\mathbf{4}$ is a weaker-binding guest, explaining why it does not noticeably retard the reaction rate.

Scheme 2: Conversion of $\mathrm{Cp} * \mathrm{H}$ into a non-competitive guest (4) to alleviate product inhibition.

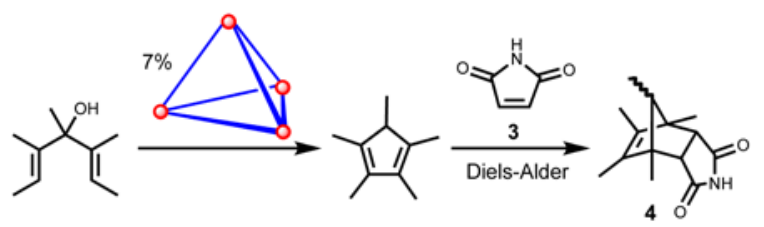

Catalysis under these conditions is quite efficient, and turnover numbers of up to 160 are achieved. The observed rate constants for the catalyzed cyclization of Z,Z substrate $\mathbf{2} \mathbf{b}$ and E,Z substrate 2c are an order of magnitude larger than that of the cyclization of E,E substrate 2a (Table 1). We find it is remarkable that the reactivity of these three substrates is so different when they differ only in stereochemistry at positions remote to the forming carbocation. Since guest exchange is fast relative to the reaction rate, the rate constants for the reaction of host-bound substrate, $k_{\text {cat }}$, were calculated from the Michaelis-Menten equation using experimentally-determined $K_{M}$ values. Substrate 2a is too weakly bound under the mixed-solvent conditions for the $K_{M}$ value to be measured, so its $k_{\text {cat }}$ value is not reported.

In order to quantify the rate acceleration attributable to catalysis, the background reaction rates of each substrate were measured in the absence of $\mathbf{1}$. The uncatalyzed reaction rates are extremely slow under these reaction conditions and strongly dependent on the substrate stereochemistry, with methyl groups in the $Z$ configuration giving the slowest reaction. This suggests that the reacting molecule must adopt a U-shaped conformation in order to react prior to or at the transition state of the rate-determining step. This compact conformation necessary for reaction is sterically disfavored by $Z$ methyl groups when compared to the linear conformation of the same molecule (Scheme 3).

Scheme 3: The linear and u-shaped conformations of substrate $\mathbf{2 b}$..

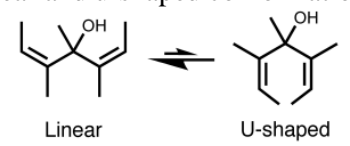

Table 1. Kinetic data for Nazarov substrates at $45{ }^{\circ} \mathrm{C}$ with maleimide added as a trapping agent.

\begin{tabular}{llllll}
\hline substrate & $\mathrm{k}_{\text {obs }}\left(\mathrm{s}^{-1}\right)$ & $\mathrm{K}_{\mathrm{M}}(\mathrm{mM})$ & $\mathrm{k}_{\text {cat }}\left(\mathrm{s}^{-1}\right)$ & $\mathrm{k}_{\text {uncat }}\left(\mathrm{s}^{-1}\right)$ & $\begin{array}{l}\text { rate acceleration } \\
\left(\mathrm{k}_{\text {cat }} / \mathrm{k}_{\text {uncat }}\right)\end{array}$ \\
\hline 2a & $5.1(1) \times 10^{-5}$ & - & - & $4.0(3) \times 10^{-8}$ & - \\
2b & $4.2(1) \times 10^{-4}$ & $42(1)$ & $1.6(1) \times 10^{-2}$ & $7.7(8) \times 10^{-9}$ & $2,100,000$ \\
2c & $1.08(2) \times 10^{-3}$ & $91(1)$ & $5.7(1) \times 10^{-2}$ & $3.3(1) \times 10^{-8}$ & $1,700,000$ \\
\hline
\end{tabular}


The rate accelerations of the catalyzed reaction over the uncatalyzed reaction are on the order of $10^{6}$; the largest measured for supramolecular catalysis by orders of magnitude. $^{14}$ This very high level of catalytic activity is reminiscent of enzymatic catalysis. The observed rate acceleration is too large to be explained only by an increase of the basicity of the bound substrate. In previous studies we observed a maximum of four orders of magnitude in equilibrium shift of protonated amines, and thousandfold rate acceleration in the hydrolysis of orthoformates. ${ }^{7,8 a}$ We propose that the additional rate enhancement in this system is due to constrictive binding in the pocket of $\mathbf{1}$, which favors both the U-shaped conformation of the substrate and the compact transition state of the electrocyclization. Constrictive binding is responsible for rate enhancements of nearly three orders of magnitude in the 1-catalyzed aza-Cope rearrangement of enammonium cations, where encapsulation preorganizes the substrate into a reactive conformation. ${ }^{9}$ We conclude that the millionfold rate enhancement in this system is due to the combination of an increase in the basicity of the alcohol functionality upon encapsulation, preorganization of the bound substrate, and stabilization of the transition state of the electrocyclic reaction. It is possible that combining the effects of constrictive binding with functional group activation represents a general strategy towards achieving similarly enhanced reactivity in supramolecular catalysis.

To probe the mechanism of this reaction, we sought to identify the encapsulated species observed during the reaction, which must be the resting state of the catalyst. The ${ }^{13} \mathrm{C}$ labeled compound $2-{ }^{13} \mathbf{C}$, prepared as a mixture of three stereoisomers, was used for this purpose. If the encapsulated species is either the dienyl cation $\mathbf{5}$ or the cyclized allyl cation 6 (Scheme 4), there should be a dramatic shift in the ${ }^{13} \mathrm{C}-\mathrm{NMR}$ resonance of the labelled carbon. ${ }^{15}$ When $2-{ }^{13} \mathbf{C}$ is encapsulated in $\mathbf{1}$, the enriched ${ }^{13} \mathrm{C}$ resonances are shifted by only a few ppm upfield compared to the unencapsulated alcohol. This is consistent with encapsulation of the alcohol $2-{ }^{13} \mathbf{C}$. The hydrophobic binding of neutral alkanes and arenes in $\mathbf{1}$ has been reported, and it is likely that the encapsulation of $\mathbf{2}$ is similar. The observation of the 1-bound substrate as the catalyst resting state is consistent with our proposed mechanism in which reversible protonation of the host-bound substrate is followed by loss of water and rate-determining electrocyclization. Future work will be directed towards deducing the full rate law of this reaction.

Scheme 4: Mechanism of the Nazarov cyclization, showing possible intermediates that are encapsulated in $\mathbf{1}$ as the resting state of the catalysis.

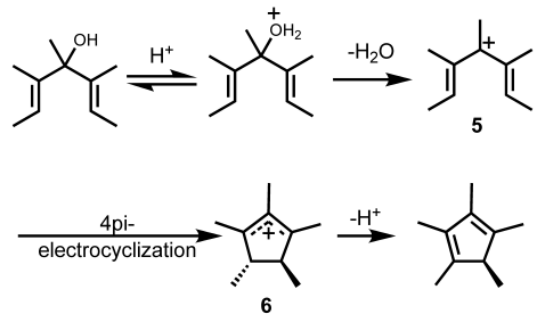

In conclusion, we have demonstrated the efficient catalysis of the Nazarov cyclization of 1,4-pentadien-3-ols by a self- assembled host in water and mixed water/DMSO. The rate of the catalyzed reaction is up to 2,100,000 times larger than that of the uncatalyzed reaction, representing the first instance of supramolecular catalysis that achieves rate enhancements comparable in size to those seen in enzymatic systems. The origin of this dramatic effect is attributed to the preorganization of the reactant and stabilization of the transition state by constrictive binding, as well as the increase in basicity of the alcohol group of the bound reactant. Preliminary mechanistic studies are consistent with this explanation.

Acknowledgment. We gratefully acknowledge financial support from the Director of the Office of Energy Research, Office of Basic Energy Sciences, Chemical Sciences Division (U.S. Department of Energy) under Contract No. DE-AC02-05CH11231, and through fellowships from the NSF (to M.D.P.) and Chevron (to C.J.H.). The authors thank Lee Bishop, Casey Brown, Jeff Mugridge, and Dr. Carmelo Sgarlatta for helpful discussions and reading of the manuscript.

Supporting Information Available: Experimental details, kinetic data, and characterization of host-guest complexes. This material is available free of charge via the Internet at http://pubs.acs.org.

\section{References}

(1) (a) Cram, D. J. Angew. Chem., Int. Ed. Engl. 1988, 27, 1009-1020. (b) Hof, F.; Craig, S.L.; Nuckolls, C.; Rebek, J. Angew. Chem. Int Ed. Engl. 2002, 41, 1488-1508.

(2) (a) Breslow, R.; Dong, S. D. Chem. Rev. 1998, 98, 1997-2012. (b) Kirby, A. J. Angew. Chem. Int. Ed. Engl. 1996, 35, 708-724. (c) Vriezema, D. M.; Aragone`s, M. C.; Elemans, J. A. A. W.; Cornelissen, J. J. L. M.; Rowan, A. E.; Nolte, R. J. M. Chem. Rev. 2005, 105, 1445-1489. (d) Yoshizawa, M.; Klosterman, J. K.; Fujita, M. Angew. Chem. Int. Ed. 2009, 48, 3418-3438.

(3) Jencks, W. P., Catalysis in Chemistry and Enzymology. McGrawHill: New York, 1969.

(4) Radzicka, A.; Wolfenden, R. A. Science, 1995, 267, 90-93.

(5) (a) Caulder, D. L.; Powers, R. E.; Parac, T. N.; Raymond, K. N. Angew. Chem. Int. Ed. 1998, 37, 1840-1843. (b) Caulder, D. L. Raymond, K. N. Acc. Chem. Res. 1999, 32, 975-982.

(6) (a) Biros, S. M.; Bergman, R. G.; Raymond, K. N. J. Am. Chem Soc. 2007, 129, 12094-12095.

(7) Pluth, M. D.; Bergman, R. G.; Raymond, K. N. J. Am. Chem. Soc 2007, 129, 11459-11467.

(8) (a) Pluth, M. D.; Bergman, R. G.; Raymond, K. N. Science 2007, 316, 85-88. (b) Pluth, M. D.; Bergman, R. G.; Raymond, K. N. Acc. Chem. Res. 2009, 42, 1650-1659.

(9) Fiedler, D.; van Halbeek, H.; Bergman, R. G.; Raymond, K. N. J. Am. Chem. Soc 2006, 128, 10240-10252.

(10) Campbell, P. H.; Chiu, N. W. K.; Deugau, K.; Miller, I. J.; Sorensen, T. S. J. Am. Chem. Soc 1969, 91, 6404-6410.

(11) (a) Threlkel, R. S.; Bercaw, J. E.; Seidler, P. F.; Stryker, J. M.; Bergman, R. G. Org. Syn., Coll. 1993, 8, 505-508. (b) King, R. B.; Bisnette, M. B. J. Organomet. Chem. 1967, 8, 287-297.

(12) Hastings, C. J.; Fiedler, D.; Bergman, R. G.; Raymond, K. N. J. Am. Chem. Soc 2008, 130, 10977-10983.

(13) (a) Kang, J. M.; Hilmersson, G.; Santamaria, J.; Rebek, J. J. Am. Chem. Soc. 1998, 120, 3650-3656. (b) Nishioka, Y.; Yamaguchi, T.; Yoshizawa, M.; Fujita, M. J. Am. Chem. Soc 2007, 129, 70007001. (c) Yoshizawa, M.; Takeyama, Y.; Kusukawa, T.; Fujita, M. Angew. Chem. Int. Ed. 2002, 41, 1347-1349.

(14) (a) Mock, W. L.; Irra, T. A.; Wepsiec, J. P.; Adhya, M. J. Org Chem. 1989, 54, 5302-5308. (b) Marinescu, L. G.; Bols, M. Angew. Chem. Int. Ed. 2006, 45, 4590-4593. (c) Very large rate accelerations were observed in the stoichiometric acylation of cyclodextrins: Trainor, G. L.; Breslow, R. J. Am. Chem. Soc. 1981, $103,154-158$

(15) Sorensen, T. S. J. Am. Chem. Soc. 1967, 89, 3782-3794. 

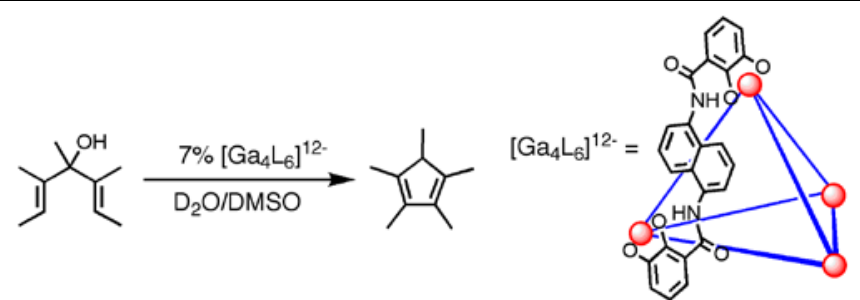

Rate enhancement of $2.1 \times 10^{6}$

A water-soluble, self-assembled, tetrahedral assembly $\mathrm{K}_{12} \mathrm{Ga}_{4} \mathrm{~L}_{6}(\mathrm{~L}=1$,5-biscatecholamide naphthalene) catalyzes the Nazarov Cyclization of 1,3-pentadienols with extremely high levels of efficiency. The catalyzed reaction proceeds at a rate over a million times faster than that of the background reaction, an increase comparable to those observed in some enzymatic systems. This catalysis operates under aqueous conditions at mild temperature and $\mathrm{pH}$ ranges, and the reaction is halted by the addition of an appropriate inhibitor. This unprecedented rate enhancement is proposed to be due to both the stabilization of protonated reaction intermediates and the effect of constrictive binding on the bound guest. 